\title{
Flexible bronchoscopy-related safety in patients with severe ARDS
}

\author{
A. Guillon ${ }^{1,2^{*}}$ (D, M.-A. Nay ${ }^{3}$ and T. Kamel ${ }^{3}$ \\ See related letter by Kalchiem-Dekel et al. https://ccforum.biomedcentral.com/articles/10.1186/s13054-018-1983-3
}

There is a lack of information regarding the safety of flexible bronchoscopy (FB) in patients with severe acute respiratory distress syndrome (ARDS). We read with great interest the letter by Kalchiem-Dekel and colleagues [1] in which they questioned the feasibility of FB in ARDS patients ventilated in the prone position. Based on retrospective ventilator-related parameters and monitoring data in seven patients, they concluded that FB is feasible in ARDS patients ventilated in the prone position, suggesting that it is safe. We would like to thank the authors for underlining this important question, but we believe that essential physiological aspects are missing in this discussion.

The insertion of the bronchoscope through the endotracheal tube (ETT) increases airway resistance by obstructing the ETT, thereby limiting respiratory flows and ultimately increasing inspiratory and expiratory pressures. If the peak inspiratory pressure is greater than the high-limit pressure set on the ventilator, the tidal volume is not fully delivered. This leads to alveolar derecruitment with well-known consequences in ARDS [2]. If the high-pressure limit is increased to maintain ventilation, the tidal volume is fully delivered but the decrease of expiratory flow results in a trapped volume within the lung that induces dynamic inflation secondary to the incomplete emptying of the lungs at the end of expiration. Hyperinflation increases the alveolar pressure, resulting in a high total PEEP and a high Pplat, possibly leading to pneumothorax in pathological lungs. This cause-effect relationship between ETT obstruction by FB and pneumothorax has been demonstrated by ventilated lung modeling in ARDS [3]. Importantly, the ventilator-measured parameters cannot capture this information during the FB procedure for two reasons. First, respiratory hold maneuvers are required to estimate the alveolar pressures delivered by the ventilator, but it seems inappropriate to perform them during FB. Second, the airway pressures measured by the ventilator during regular hold maneuvers hardly reflect the alveolar pressures because the ETT is obstructed by the bronchoscope. The airway pressures should be measured after the obstruction. Using a manometer placed on the suction channel of the bronchoscope could be an alternative solution [4]. In the individual situations reported by the authors, an immediate increase of $+7 \mathrm{cmH} 2 \mathrm{O}$ of alveolar pressure is expected when a FB tube is inserted in the ETT with a $1.7 \mathrm{~mm}$ difference in diameters $[1,5]$. In conclusion, FB induces unnoticed parenchymal overdistention and derecruitment, which may worsen existing injury; the evolution of the oxygenation parameters could be the tip of the iceberg in terms of FB-related safety in severe ARDS.

\section{Authors' response}

\section{Or Kalchiem-Dekel, Ashutosh Sachdeva, Jean Jeudy, Edward M. Pickering and Carl B. Shanholtz}

We thank Drs. Guillon, Nay, and Kamel for their interest in our Letter to the Editor [1] and their insightful comments on the physiological consequences of ETT obstruction due to insertion of the bronchoscope in patients with

\footnotetext{
* Correspondence: antoine.guillon@univ-tours.fr

${ }^{1}$ CHRU de Tours, Service de Médecine Intensive Réanimation, Tours, France ${ }^{2}$ INSERM, Centre d'Etude des Pathologies Respiratoires, U1100 Tours, France Full list of author information is available at the end of the article
}

ARDS. The authors' work on the subject has enhanced our understanding on bronchoscopy in ARDS patients $[4,5]$. We acknowledge the importance of clearance between bronchoscope and ETT (in our series it was at least $1.7 \mathrm{~mm}$ ) to minimize resistance, reduction of minute ventilation, and development of excessive end-expiratory pressure and resultant alveolar overdistention. As outlined 
by Dr. Guillon and colleagues, these can have potential adverse consequences.

Our data are limited by the retrospective nature of the study and the lack of intra-procedural pressure measurements. The primary objective of our case series was to highlight the feasibility and possible benefit of secretion clearance in ARDS patients while in the prone position. We point out that all patients who underwent bronchoscopy while in the prone position in our case series had mild to moderate ARDS by the Berlin definition [6], whereas the lung models used by Dr. Guillon and colleagues were for severe ARDS [5]. Additionally, unlike static experimental models, real-life bronchoscopy is a dynamic process. To reduce the risk of derecruitment or increase in end-expiratory pressure, all bronchoscopy procedures in our series were performed in brief sequential cycles and suctioning was performed in accordance with recommendations made by the American Association for Respiratory Care regarding mechanically ventilated patients [7]. In the report by Nay et al., however, a different methodology was employed [5]. Furthermore, in our series there were no instances of post-procedure pneumothorax, a potential complication of breath stacking. This is likely a result of two factors: (a) the high-pressure limit was not increased; (b) application of suctioning may have ameliorated end-expiratory pressure build-up.

Finally, data regarding optimal performance of bronchoscopy in patients with ARDS is scarce. We read with great interest the report by Nay et al. describing a novel flexible bronchoscope designed specifically for preservation of lung protective ventilation in mechanically ventilated patients [4]. We hope that this innovation, in conjunction with better designed studies, will provide us with information needed to develop best practice guidelines for bronchoscopy procedures in patients with ARDS and possibly decrease risk of procedure-related complications.

\section{Abbreviations}

ARDS: Acute respiratory distress syndrome; ETT: Endotracheal tube;

FB: Flexible bronchoscopy

\section{Acknowledgements}

We would like to thank Elise Symer for English editing.

\section{Funding}

This research received no specific grant from any funding agency in the public, commercial, or not-for-profit sectors.

\section{Authors' contributions}

AG coordinated the redaction of the manuscript. MAN and TK helped to draft the manuscript. All authors read and approved the final manuscript.

\section{Competing interests}

The authors declare that they have no competing interests.

\section{Author details}

${ }^{1}$ CHRU de Tours, Service de Médecine Intensive Réanimation, Tours, France. 2INSERM, Centre d'Etude des Pathologies Respiratoires, U1100 Tours, France. ${ }^{3} \mathrm{CHRO}$ d'Orléans, Service de Médecine Intensive Réanimation, Orléans,

France.

Received: 4 April 2018 Accepted: 16 May 2018

Published online: 21 June 2018

\section{References}

1. Kalchiem-Dekel O, Shanholtz CB, Jeudy J, Sachdeva A, Pickering EM Feasibility, safety, and utility of bronchoscopy in patients with ARDS while in the prone position. Crit Care. 2018;22:54.

2. Lapinsky SE, Mehta S. Bench-to-bedside review: recruitment and recruiting maneuvers. Crit Care. 2005:9:60-5.

3. Nay M-A, Mankikian J, Garot D, Guillon A. Investigation of a cause-effect relationship between flexible bronchoscopy and pneumothorax in patients with severe acute respiratory distress syndrome. Eur J Anaesthesiol. 2015;32:896-8.

4. Nay M-A, Auvet A, Mankikian J, Herve V, Dequin P-F, Guillon A. Evaluation of a flexible bronchoscope prototype designed for bronchoscopy during mechanical ventilation: a proof-of-concept study. Anaesthesia. 2017;72:719-28.

5. Nay M-A, Mankikian J, Auvet A, Dequin P-F, Guillon A. The effect of fibreoptic bronchoscopy in acute respiratory distress syndrome: experimental evidence from a lung model. Anaesthesia. 2016:71:185-91.

6. Ranieri VM, Rubenfeld GD, Thompson BT, Ferguson ND, Caldwell E, Fan E, Camporota L, Slutsky AS. Acute respiratory distress syndrome: the Berlin Definition. JAMA. 2012;307(23):2526-33.

7. Strickland SL, Rubin BK, Haas CF, Volsko TA, Drescher GS, O'Malley CA. AARC clinical practice guideline: effectiveness of pharmacologic airway clearance therapies in hospitalized patients. Respir Care. 2015;60(7):1071-7.

\section{Publisher's Note}

Springer Nature remains neutral with regard to jurisdictional claims in published maps and institutional affiliations. 